\title{
INVENTAIRE BIBLIOGRAPHIQUE DES ALGUES BENTHIQUES DU LITTORAL MAROCAIN. I. CHLOROPHYCEAE ET PHAEOPHYCEAE
}

\author{
RIADI Hassane \& KAZZAZ Mohamed
}

RÉSUMÉ. Inventaire préliminaire des algues benthiques du littoral marocain. I. Chlorophyceae et Phaeophyceae. L'inventaire bibliographique des Chlorophyceae et Phaeophyceae marines benthiques du littoral marocain a révélé 213 espèces dont 93 Chlorophyceae ( 6 ordres, 15 familles et 31 genres) et 110 Phaeophyceae (11 ordres, 20 familles et 50 genres)

Mots clés. Littoral marocain, algues marines benthiques, Chlorophyceae, Phaeophyceae.

ABSTRACT. A preliminary check-list of Moroccan seaweed. I. Chlorophyceae and Phaeophycae. A bibliographical inventory of Chlorophyceae and Phaeophyceae from Moroccan coast has revealed 213 species whose 93 Chlorophyceae (6 orders, 15 families and 31 genus) and 110 Phaeophyceae (11 orders, 20 families and 50 genus).

Key words. Seaweed, Moroccan coast, Chlorophyceae, Phaeophyceae.

\section{INTRODUCTION}

Les premières études de la phycoflore benthique du littoral marocain remontent à la fin du siècle dernier, avec le travail de P. K. Schousboe sur la côte tangeroise dont le résultat a été révisé et publié par Bornet (1892). Ce travail présente un premier aperçu sur les algues marines de la région, de même celui de Debray (1897).

Vers le début du siècle de nouveaux travaux sont apparus dont: Hariot (1909/11) qui s'est intéressé à la côte marocaine en général; De Buen (1913) et Sauvageau (1913) qui ont travaillé sur les Fucales et Laminariales de la façade méditerranéenne.
Dès la moitié de ce siècle, les côtes marocaines ont suscité un intérêt particulier de la part de plusieurs auteurs tels:

-Werner (1948/62) qui a présenté quelques réflexions sur la zonation des algues sur le littoral marocain;

-Gattefosse et al. (1935), Dangeard (1937/57), Feldmann (1955), Gayral (1957/61), Esmiole (1962), Cavassillas (1963), Salaheddine (1982), Tahi (1983), Ouahi (1987), Berday (1989), Riadi (1989) qui ont étudié la phycoflore benthique du littoral atlantique; -Lozano Cabo (1953), Conde (1984), Kazzaz (1989); Gonzalez et Conde (1991/95) dont les traveaux ont porté spécialement sur la côte méditerranéenne. 
L'analyse de cet aperçu bibliographique montre que les données sur la phycoflore de nos côtes sont très fragmentaires et dispersées; la nécessité de les regrouper s'impose d'emblée.

L'assemblage de ces résultats pourrait avoir une grande valeur bibliographique pour des études ultérieures et c'est cet aspect qui nous a incité à aborder le présent travail qui représente une première partie de l'inventaire préliminaire concernant la phycoflore marine benthique pluricellulaire ou macroscopique appartenant à la classe des Chlorophyceae et à celle des Phaeophyceae des étages littoraux (Supralittoral, Médiolittoral, Infralittoral et Circalittoral) du système phytal (Pérès et Piccard 1964) du littoral marocain.

\section{METHODOLOGIE}

Pour la nomenclature nous avons adopté celle utilisée par Dangeard (1949); Hamel (1930 et 1939); Gayral (1958 et 1966) avec les réactualisations apportées successivement par Boudouresque et al. (1984), PerretBoudouresque \& Seridi (1989) sur les deux classes, Burrows (1991) et Gallardo et al. (1993) sur les Chlorophyceae; Ribera et al. (1992) sur les Phaeophyceae et enfin FloresMoya et al. (1995a et 1995b) respectivement sur les Phaeophyceae et les Chlorophyceae.

Pour chacune des classes représentées, les ordres, les familles, les genres et les espèces sont rangés par ordre alphabétique. Le nom retenu de chaque espèce a été suivi par ses synonymes les plus courants. Les chiffres précédant chaque espèce renvoient aux références numérotées en bibliographie et entre parenthèses la localisation géographique (fig. 1) signalée par l'auteur. En astérix, d'autres régions où nous avons rencontré l'espèce et les chiffres en exposant correspondent aux notes.

En général les variétés et les formes n'ont pas été prises en considération.

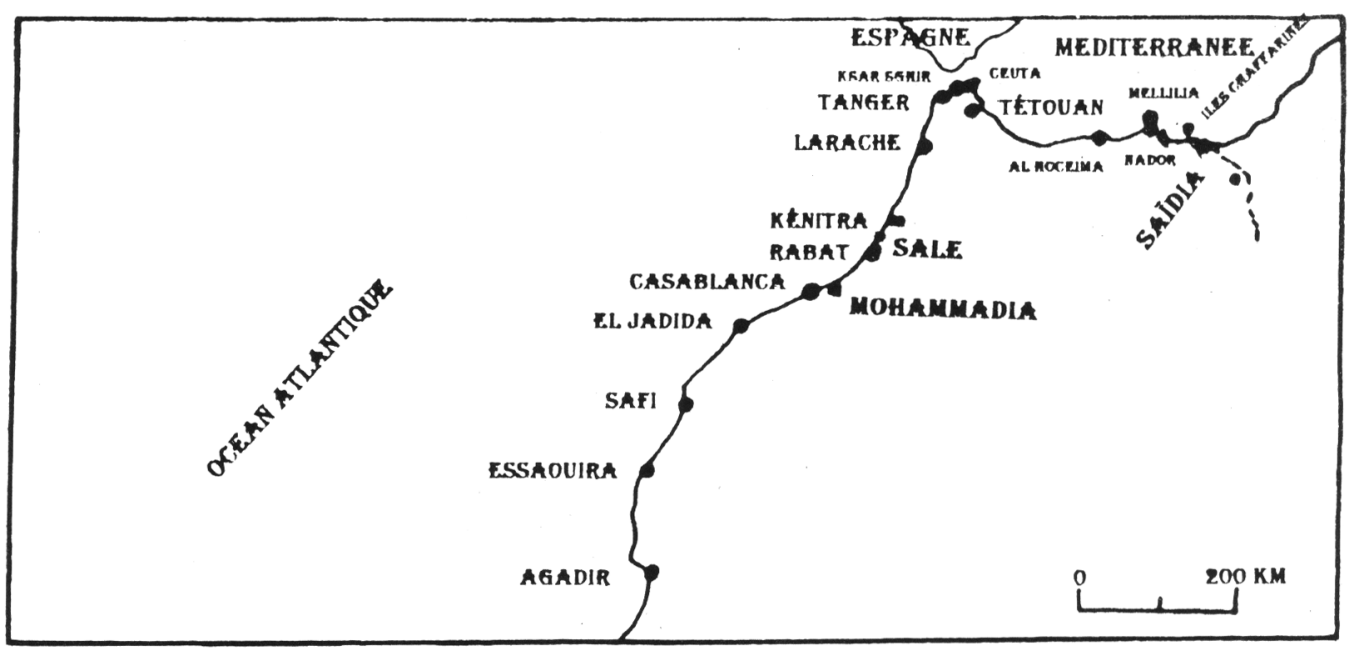

Figure 1. Carte de répartition des localités signalées. Map of situation of the signalled localities. 


\section{LISTE DES TAXA INVENTORIES}

Abréviations utilisées:

Côte méditerranéenne; Ceuta (Ceu), Tetouan (Tet), Al Houceima (Alh), Nador (Nad), Mellilia (Mel), Saïdia (Sai), les Iles Chaffarines (Ich), Mediterranée (Med).

Détroit de Gibraltar: Ksar Sghir (Ksg).

Côte Atlantique; Tanger (Tan), Larache (Lar), Salé (Sal), Kenitra (Ken), Rabat (Rab), Mohammadia (Moh), Casablanca (Cas), El Jadida (Elj), Safi (Saf), Essaouira (Ess), Agadir (Aga), Atlantique (Atl).

CHLOROPHYCEAE Wille s. 1 .

I-Acrosiphoniales Jónsson

Acrosiphoniaceae Jónsson

Urospora Areschoug

U. leata (Thuret) Boergesen

a) 4, 34(Tan); 12, 21(Rab, Tan); 38(Tet, Ksg); 40(Moh)

\section{II-Byopsidales Schaffner}

\section{Byopsidaceae Bory}

Bryopsidella J. Feldmann

B. neglecta (Berthold) Rietema

$=$ Derbesia neglecta Berthold

a) 12, 21(Aga); 31(Nad, Mel, Alh); 40(Moh)

Bryopsis Lamouroux

B. adriatica (J. Agardh) Meneghini

= B. cupressina Lamouroux var. adriatica

J. Agardh

a) 4, 12, 21(Rab, Sal)

B. corymbosa J. Agardh

a) 1(Elj); 2(Saf, Ess); 7(Rab); 12, 21(Rab, Ess); 23(Atl); 31(Nad, Mel); 38(Tet, Ksg); $48($ Sal)
B. duplex De Notaris

$=$ B. balbisiana Lamouroux sensu

Feldmann

= B. disticha (J. Agardh) Kützing

a) 1(Elj), 4, 34(Tan); 7(Rab); 12, 21(Rab, Cas); 23(Atl, Med); 31(Alh, Nad, Mel); 40(Moh);48(Sal); (Tet)*

B. feldmannii Gallardo et Furnari

$=$ B. cupressoides Kützing sensu J. Feldmann

a) 4, 34(Tan); 31(Mel)

B. hypnoides Lamouroux

= B. monoica Berthold ex Funk

a) $31(\mathrm{Alh}, \mathrm{Nad}, \mathrm{Mel})$; 38(Tet, Ksg); 40(Moh)

B. muscosa Lamouroux

a) 4, 34(Tan); 12, 21(Rab, Cas); 31(Alh, Nad, Mel), 40(Moh)

B. pennata Lamouroux

a) $12,21(\mathrm{Rab})$

B. plumosa (Hudson) C. Agardh

a) 1(Elj), 7, 16(Rab); 12, 21(Rab, Cas); 23(Atl, Med); 28, 32(Nad, Mel); 31(Med); 38(Tet, Ksg); 40(Moh); 48(Sal); (Tan)*; (Lar)*

\section{Caulerpaceae kützing}

Caulerpa Lamouroux

C. prolifera (Forsskäl) Lamouroux

a) $28,31(\mathrm{Nad}, \mathrm{Mel})$

C. racemosa (Forsskäl) J. Agardh a) 9 (Tan)

Codiaceae Kützing

Codium Stackhouse

C. adhaerens (Cabrera) C. Agardh

a) 1, 17(Elj); 7(Rab); 22(Ess); 23(Atl, Med); 31(Ceu, Alh, Nad, Mel); 38(Tet, Ksg); 40(Moh); 48(sal); (Tan, )*; (Lar)*

C. bursa (Linnaeus) C. Agardh

a) 4, 34(Tan); 31(Nad, Mel); 38(Tet, Ksg) 
C. decorticatum (Woodward) M. Howe $=\mathrm{C}$. elongatum (Turner) C. Agardh a) 1(Elj);2(Saf, Ess); 4, 34(Tan); 7, 16(Rab, Cas); 12, 21(Rab à Aga); 23(Atl, Med); 31(Nad, Mel); 38(Tet, Ksg); 40(Moh); 48(Sal), (Tan)*, (Lar)*

C. effusum (Rafinesque) Delle Chiaje = C. difforme Kützing

a) 1(Elj); 4, 34(Tan); 7, 23(Rab ); 12, 21(Rab, Aga, ); 31(Alh, Nad, Mel); 32(Nad); 38(Tet, Ksg); 40(Moh)

C. fragile (Suringar) Hariot
a) $31(\mathrm{Mel})$

C. tomentosum Stackhouse

a) 1(Elj); 2(Saf, Ess); 4, 34(Tan); 7, 12, 16, 21(Rab); 23(Atl, Med); 31(Ceu, Nad, Mel); 38(Tet, Ksg); 40(Moh); 48(Sal), (Tan)*

C. vermilara (Olivi) Delle Chiaje

a) $31(\mathrm{Nad}, \mathrm{Mel}, \mathrm{Alh})$

\section{Derbesiaceae Hauck}

\section{Derbesia Solier}

D. tennuissima (De Notaris) P. L. et H. M. Crouan ${ }^{(3)}$ (Stade sporophytique de Halicystis parvula)

a) 4, 34(Tan); 7(Rab); 12, 21(Rab, Aga); 23(Atl, Med); 28, 32(Nad, Mel); 31(Med); 38(Tet, Ksg); 40(Moh); 48(Sal)

\section{Halicystis Areschoug}

H. parvula Schmidt in Murrey

a) 7, 16(Rab); 12, 21(Rab); 23(Atl, Med); 31(Nad, Mel); 38(Tet); 40(Moh); 48(Sal)

Pedobesia MacRaild et Womersley

P. lamourouxii (J. Agardh) J. Feldmann et al. = Derbesia lamourouxii (J. Agardh) Solier a) 7(Rab); 12, 21(Rab, Cas); 23(Atl, Med); 28, 31, 32(Nad, Mel); 38(Tet); 40(Moh)

P. solieri J. Feldmann ex Abelard et Knoepffler

a) $31(\mathrm{Nad}, \mathrm{Mel}, \mathrm{Sai})$
Udoteaceae J. Agardh

Flabellia Reichenbach

F. petiolata (Turra) Nizamuddin = Udotea petiolata (Turra) Boergesen

a) 31 (Nad, Mel, ICh)

Halimeda Lamouroux

H. tuna (J. Ellis et Solander) Lamouroux

a) 4, 34(Tan); 31(Ich)

Pseudochlorodesmis Boergesen

P. furcellata (Zanardini) Boergesen

a) 38(Tet, Ksg); 40(Moh)

\section{III-Cladophorales Haeckel}

\section{Cladophoraceae Wille}

Chaetomorphora Kützing

C. aerea (Dillwyn) Kützing

a) 1(Elj); 2(Saf, Ess); 4, 34(Tan); 7, 16(Rab); 12, 21(Rab); 23(Atl, Med); 38(Tet, Ksg); 40(Moh); 48(Sal)

C. gracilis Kützing
a) 31 (Alh, Nad, Mel)

C. linum (O. F. Müller) Kützing

a) 21(Cas); 28, 32(Nad, Mel); 31(Med)

C. mediterranea Kützing

= C. capillaris (Kützing) Boergesen

a) 1(Elj); 4, 34(Tan); 12, 21(Ess); 38(Tet); 40(Moh)

C. pachynema Montagne

a) 1(Elj), 7(Rab); 12, 21(Rab); 23(Atl, Med); 38(Tet, Ksg); 40(Moh); 48(sal)

Cladophora Kützing

C. albida (Nees) Kützing

a) = C. hamosa (Kützing) Kützing; 2(Saf, Ess); 31(Ich)

C. coelothrix Kützing = C. repens (J. Agardh) Harvey

a) 21(Cas);28(Nad, Mel);31(Med);38(Tet, Ksg) 
C. dalmatica Kützing

a) 28, 32(Nad, Mel); 31(Med); 38(Tet, Ksg)

C. echinus (Biasoletto) Kützing

a) 28, 32(Nad, Mel); 31(Alh, Nad, Mel)

C. globulina (Kützing) Kützing

a) 28, 32(Nad, Mel); 31(Alh, Nad, Mel)

C. hirta Kützing

a) 40 (Moh)

C. hutchinsiae (Dillwyn) Kützing

a) 2(Saf, Ess); 31 (Alh, Nad, Mel)

C. laetevirens (Dillwyn) Kützing

a) 40 (Moh)

C. lehmanniana (Lindenberg) Kützing

$=\mathrm{C}$. ramulosa Meneghini

=C. utriculosa Kützing

a) 12, 21(Rab, Cas); 16(Rab); 28, 32(Nad, Mel); 31(Alh, Nad, Mel)

C. pellucida (Hudson) Kützing

a) 1 (Elj); 2(Saf, Ess); 4, 34(Tan); 7, 16(Rab); 12, 21(Rab, Cas); 23(Atl, Med); 28, 32(Nad, Mel); 48(Sal)

C. prolifera (Roth) Kützing

a) 1(Elj); 7(Rab); 12, 21(Rab, Cas); 23(Atl, Med); 28(Nad, Mel); 31(Tet, Alh, Nad, Mel); 38(Tet, Ksg); 40(Moh); 48(sal)

C. punica (Hudson) Kützing

a) 40 (Moh)

C. rupestris (Linnaeus) Kützing

= C. ramosissima (Drapanaud ex Kützing) Kützing

a) 1(Elj); 2(Saf, Ess); 4, 34(Tan); 7(Rab); 12, 21(Rab, Cas); 23(Atl); 31(Alh, Nad, Mel); 40(Moh); 48(sal)

C. sericea (Hudson) Kützing $=$ C. rudolphiana (C.Agardh) Kützing

a) 4, 34(Tan); 21(Cas); 31 (Alh, Nad, Mel); 38(Tet)

C. vadorum (Areschoug) Kützing

a) $28,31,32$ (Alh, Nad, Mel)
C. vagabunda (Linnaeus) Hoek

a) $28(\mathrm{Nad}, \mathrm{Mel}) ; 31(\mathrm{Alh}, \mathrm{Nad}, \mathrm{Mel})$

Cladophoropsis Bory

C. membranaceus (C. Agardh) Boergesen

a) 21 (Cas)

\section{Rhizoclonium Kützing}

R. tortuosum (Dillwyn) Kützing $=\mathrm{R}$. riparium (Roth) Harvey

$=\mathrm{R}$. kochianum Kützing

$=\mathrm{R}$. kerneri Stockmayer

a) 4, 34(Tan); 12, 21(Ess); 28, 32(Nad, Mel); 31(Med)

\section{Siphonocladaceae Schmitz}

Valonia C. Agardh

V. macrophysa Kützing

a) 31 (Alh, Nad, Mel)

V. utricularis (Roth) C. Agardh

a) 1 (Elj); 4, 34(Tan); 7, 16(Rab); 12, 21(Rab, Cas); 23(Atl, Med); 28, 32(Nad, Mel); 31(Med); 38(Tet, Ksg); 40(Moh)

Ventricaria Olsen et J. A. West

V. ventricosa (J. Agardh) Olsen et J. A. West = Valonia ventricosa J. Agardh

a) 31 (ICh)

\section{Dasycladales Bessey}

Dasycladaceae Kützing

Dasycladus C. Agardh

D. vermicularis (Scopoli) Krasser = D. clavaeformis (Roth) C. Agardh

a) $31(\mathrm{Nad}, \mathrm{Mel})$

\section{Polyphysaceae Kützing}

Acetabularia Lamouroux

A. acetabulum (Linnaeus) Silva

$=$ A. mediterranea Lamouroux

a) $28(\mathrm{Nad}, \mathrm{Mel}) ; 31(\mathrm{Nad}, \mathrm{Mel}, \mathrm{ICh})$

A. calyculus Lamouroux

a) $31(\mathrm{Nad}, \mathrm{Mel})$ 
Polyphysa Lamarck

P. parvula (Solms) Schnetter et Bula-Mayer $=$ Acetabularia moebii Solms

$=$ A. parvula Solms

a) $31(\mathrm{Nad}, \mathrm{Mel})$

\section{Ulotrichales Borzi}

\section{Chaetophoraceae Greville}

\section{Enthocladia Reinke}

E. viridis Reinke

$=$ Phaeophila viridis (Reinke) Burrows

= Endoderma viride (Reinke) Lagerheim

a) 12,$21 ; 31(\mathrm{Nad}, \mathrm{Mel}, \mathrm{Alh})$

\section{Phaeophila Hauck}

P. dendroides (P. L. et H. M. Crouan) Batters $=\mathrm{P}$. divaricata $($ Huber) Nielsen

a) $31(\mathrm{Nad}, \mathrm{Mel}, \mathrm{Alh})$

\section{Pringsheimiella Höhnel}

P. scutata (Reinke) Hönel ex. Marchewianka a) 12, 21(Aga); 31(Nad, Mel, ICh)

\section{Pseudopringsheimia Wille}

P. confluens (Rosenvinge) Wille = Ulvella confluens Rosenvinge
a) 31 (Ich)

Ulvella P. L. et H. M. Crouan

U. setchellii P. Dangeard

a) 12,21 (Cas, Ess)

\section{Ulotrichaceae Kützing}

\section{Ulothrix Kützing}

U. flacca (Dillwyn) Thuret

a) 7(Rab); 12, 21(Cas); 31(Tet); 48(sal)

U. subflaccida Wille

a) 31 (Alh, Nad)

VI-Ulvales Blackman et Tansley

Monostromataceae Kunieda

Blidingia Kylin
B. marginata (J. Agardh) P. Dangeard

= Enteromrpha marginata J. Agardh

a) $12,21(\mathrm{Cas}, \mathrm{Elj}) ; 28,32(\mathrm{Nad}, \mathrm{Mel})$;

31(Alh, Nad, Mel); 40(Moh)

B. minima (Nägeli ex Kützing) Kylin

a) 1(Elj); 7(Rab); 12, 21(Rab, Ess); 23(Atl);

28(ICh); 38(Tet, Ksg); 40(Moh); 48(Sal)

\section{Monostroma Thuret}

M. grevillei (Thuret) Wittrock

= Ulvopsis grevillei (Thuret) P. Dangeard

a) 40 (Moh)

M. obscurum (Kützing) J. Agardh

a) 2(Saf, Ess); 12, 21(Tan, Ess)

M. oxycoccum (Kützing) Thuret

a) $7(\mathrm{Rab}) ; 12,21(\mathrm{Elj})$

\section{Percursariaceae Bliding}

\section{Percursaria Bory}

P. percusa (C. Agardh) Rosenvinge a) 21 (Cas, Elj)

Ulvaceae Lamouroux ex Dumortier

Enteromorpha Link

E. clathrata (Roth) Greville

a) 1(Elj); 4, 34(Tan); 7(Rab); 12, 21(Rab, Ess); 23(Atl, Med); 28, 32(Nad, Mel); 31(Alh, Nad, Mel), 38(Tet, Ksg); 40(Moh); 48(sal)

E. compressa (Linnaeus) Nees

a) 4, 34(Tan); 7(Rab); 12, 21(Rab, Cas, Ess); 23; 28, 32(Nad, Mel); 38(Tet, Ksg); 40(Moh); 48(sal); (Tan, Lar)*

E. fasciculata P. Dangeard
a) $12,21(\mathrm{Rab})$

E. flabellata P. Dangeard
a) 2(Saf, Ess); 7, 16(Rab)

E. flexuosa (Wulfen) J. Agardh $=$ E. lingulata J. Agardh

a) 4, 34(Tan); 12, 21, 22(Ess, Aga); 28, 32(Nad, Mel); 31(Med) 
E. intestinalis (Linnaeus) Nees

a) $7(\mathrm{Rab}) ; 12,21$ (Rab, Cas, Ess); 23; 27(Ess); 28, 32(Nad, Mel) ; 31(Med); 38(Tet); 40(Moh); 48(sal)

E. kylinii Bliding
a) $31(\mathrm{Nad}, \mathrm{Mel})$

E. linza (Linnaeus) J. Agardh

a) 1 (Elj);12, 21(Rab, Ess); 16(Rab); 23 ;27(Ess); 28, 32(Nad, Mel); 31(Medl), 38(Tet); 40(Moh); 48(sal); (Tan, Lar)*

E. multiramosa Bliding

a) $28(\mathrm{Nad}, \mathrm{Mel}) ; 31(\mathrm{Med})$

E. muscoides (Clemente) Cremades

= E. ramulosa (Smith) Carmichael

= E. crinita (Roth) J. Agardh

a) 1(Elj); 2(Saf, Ess); 4, 34(Tan); 7, 16(Rab); 12, 21(Rab à Ess); 23(Atl, Med); 31(Nad, ICh); 38(Tet, Ksg); 40(Moh); 48(sal); (Tan)*

E. prolifera (O.F. Müller) J. Agardh

a) $28(\mathrm{Nad}, \mathrm{Mel}) ; 31(\mathrm{Tet}, \mathrm{ICh})$

E. scopulorum Villot
a) $7(\mathrm{Rab})$

E. simplex (Vinogradova) Koeman et Hoek
a) 31 (Tet, ICh)

E. torta (Mertens) Reinbold

a) 4, 34(Tan); 28, 32(Nad, Mel)

E. tuberculosa P.Dangeard
a) $12,21(\mathrm{Rab})$

Ulva Linnaeus

U. bifrons Ardré

a) 2(Saf, Ess)

U. cribosa (J. Agardh) Bornet

a) 4, 34(Tan); 31(Ceu)

U. curvata (Kützing) De Toni

a) 31 (Ich)

U. elegans P. Dangeard

a) $7(\mathrm{Rab})$
U. fasciata Delile

a) 7, 16(Rab); 12(Tan); 23(Cas); 40(Moh);

48(Sal); (Tan)*

U. lactuca (C. Agardh) Le Jolis (1)

a) 1(Elj); 2(Saf, Ess); 7, 12, 16, 21(Rab); 9(Tan); 23(Atl), 27(Ess); 38(Tet, Ksg); 40(Moh); 48(Sal)

U. rigida C. Agardh

a) 2(Saf, Ess); 7, 12, 21(Rab); 27(Ess); 28(Nad, Mel), 31(Med); 48(Sal)

Ulva olivascens P. Dangeard

a) 28, 32( Nad, Mel); 31(Alh, Nad, Mel); 48 (Sal)

U. schousboei Bornet ${ }^{(2)}$

a) 4(Tan)

\section{PHAEOPHYCEAE Bory}

\section{Chordariales Setchell et Gardner}

\section{Chordariaceae Greville}

Liebmannia J. Agardh

L. leveillei J. Agardh

a) 4, 35(Tan)

Mesogloia C. Agardh

M. vermiculata (Smith) S. F. Gray

a) 38 (Tet, Ksg)

Sauvageaugloia Hamel ex Kylin

S. griffithsiana (Greville) Hamel ex Kylin = Mesogloia griffithsiana C. Agardh a) 4, 35(Tan)

\section{Corynophlaeaceae Oltmanns}

Leathesia S. F. Gray

L. difformis (Linnaeus) Areschoug = L. marina Endlicher

a) 4, 35(Tan); 7(Rab); 12, 21(Rab, Cas); 38(Tet, Ksg); 48(Sal)

Myriactula Kuntze

M. rivulariae (Suhr) J. Feldmann 
$=$ Gonodia rivulariae (Suhr) Hamel

a) 4,35 (Tan)

\section{Elachistaceae Kjellman}

\section{Elachista Duby}

E. flaccida (Dillwyn) Areschoug

a) 4, 35(Tan); 7(Rab); 12, 21(Cas, Elj); 23(Alt); 48(sal)

E. fucicola (Velley) Areschoug

a) 2(Saf, Ess); 4, 35(Tan)

\section{Leptonematella Silva}

L. fasciculata (Reinke) Silva $=$ Leptonema fasciculatum Reinke

a) 4, 35(Tan)

\section{Lithosiphon Harvey}

L. laminariae (Lyngbye) Harvey

a) 12, 21 (Cas, Ess)

\section{Myrionemataceae Nägeli}

Myrionema Greville

M. strangulans Greville

$=\mathrm{M}$. vulgare Thuret

= M. vulgare Thuret var. maculaeformis Kützing

a) 38 (Tet, Ksg)

\section{Cutleriales Kjellman}

Cutleriaceae Hauck

Aglaozonia Zanardini

A. melanoidea (Schousboe) Sauvageau (3) (Stade sporophytique de Cutleria adspersa)

a) 4, 35(Tan)

A. parvula (Greville) Zanardini (4) (Stade sporophytique de Cutleria multifida)

a) 4, 35(tan); 31(Alh, Nad, Mel, Sai)

\section{Cutleria Greville}

C. adspersa (Roth) De Notaris ${ }^{(3)}$ (gamétophyte d'Aglaozonia melanoidea)

= Zonaria adspersa C. Agardh

a) 1(Elj); 7(Rab); 12, 21(Rab, Ess, Aga);
23(Atl, Med); 31(Alh, Nad, Mel, Sai); 38(Tet, Ksg); 40(Moh); 48(Sal)

C. multifida (Smith) Greville ${ }^{(4)}$ (gamétophyte d'Aglaozonia parvula)

a) $31($ Alh, Nad); 38(Tet)

Zanardinia Nardo ex Crouan frat.

Z. prototypus (Nardo) Nardo

= Zanardinia collaris (C. Agardh) Crouan et Crouan

a) 4 , 35(Tan); 21(Cas)

III. Desmarestiales Setchell et Gardner

Desmarestiaceae (Thuret) Kjellman

Desmarestia Lamouroux

D. ligulata (Lightfoot) Lamouroux

a) 12, 21(Ess); 23(Atl)

D. tingitana nov. nom in Hamel ${ }^{(5)}$

a) 4, 35(Tan)

IV. Dictyosiphonales Setchell et Gardner

Punctariaceae (Thuret) Kjellman

Asperococcus Lamouroux

A. bullosus Lamouroux

$=$ A. turneri (Smith) Hooker

a) 4, 35(Tan); 28, 31, 32(Nad, Mel); 38(Tet, $\mathrm{Ksg}$ )

A. compressus (Griffiths) ex Hooker =Haloglossum compressum (Griffiths) Hamel

a) 4, 35(Tan)

\section{Dictyotales Kjellman}

Dictyotaceae Lamouroux ex Dumortier

Dictyopteris Lamouroux

D. polypodioides (D.C.) Lamouroux = D. membranacea (Stackhouse) Batters

a) 1(Elj); 2(Saf, Ess); 4, 35(Tan); 7(Rab); 
9(Tan); 11, 12, 21(Rab à Ess); 23(Atl, Med); 31(Med); 38(Tet, Ksg); 40(Moh); 48(Sal); 50

Dictyota Lamouroux

D. dichotoma (Hudson) Lamouroux = Zonaria dichotoma C. Agardh

a) 1(Elj); 2(Saf, Ess); 4, 35(Tan); 7, 50(Rab); 11, 12, 21, 22(Rab à Ess); 23(Atl, Med); 28, 32(Nad); 31(Med); 38(Tet, Ksg); 40(Moh); 48(Sal)

D. linearis (C. Agardh) Greville

$=$ D. divaricata Lamouroux

= Zonaria linearis $\mathrm{C}$. Agardh

a) 4, 35(Tan); 12, 21(cas); 31( Nad, Mel)

Dilophus J. Agardh

D. fasciola (Roth) Howe

a) 1(Elj); 4, 35(Tan); 7, 16(Rab);12, 21(Cas); 23(Atl, Med); 31(Alh, Nad, Mel); 32(Nad); 38(Tet, Ksg); 40(Moh); 50

D. spiralis (Montagne) Hamel = D. ligulatus (Kützing) Feldmann

a) 1 (Elj); 4, 35(Tan); 12, 21(Rab à Aga); 23(Atl, Med); 31(Med); 32(Nad); 38(Tet, Ksg); 40(Moh)

\section{Padina Adanson}

P.pavonica (Linnaeus) Lamouroux $=$ P. pavonia (Linnaeus) Gaillon a) $1(\mathrm{Elj}) ; 7(\mathrm{Rab}) ; 4,35(\mathrm{Tan}) ; 11,12,21$ (Rab à $\mathrm{Aga}) ; 23(\mathrm{Atl}, \mathrm{Med}) ; 28,32(\mathrm{Nad})$; 31(Med); 38(Tet, Ksg); 40(Moh)

\section{Spatoglossum Kützing}

S. areschougii J. Agardh

a) 4, 35(Tan); 12, 21(Rab);

S. solieri (Montagne) Kützing

a) 7(Rab); 12, 21(Rab à Cas); 23(Atl); 31(Alh); 40(Moh)

Taonia J. Agardh

T. atomaria (Woodward) J. Agardh

a) 4, 35(Tan); 7(Rab); 12, 21(Rab à Saf); 23(Atl, Med); 28, 32(Nad); 31(Alh, Nad, $\mathrm{Mel}) ;(\mathrm{Ksg})^{*}$

Zonaria Drapanald
Z. tournefortii (Lamouroux) Montagne a) 4, 35(Tan); 7(Rab); 12, 21, 48(Sal); 23(Rab, Med); 28, 32(Nad); 31(Alh, Nad, Mel); 40(Moh); (Tet, Ksg)*

\section{Ectocarpales Setchell et Gardner}

Ectocarpaceae C. Agardh

\section{Acinetospora Bornet}

A. crinita (Carmichael) Sauvageau = A. vidovichii (Meneghini) Sauvageau

a) $31(\mathrm{Nad}, \mathrm{Mel})$

A. pusilla (Griffiths) Bornet

a) 38 (Tet)

Bachelotia Bornet

B. fulvescens (Schousboe) Bornet

a) 1 (Elj); 4, 35(Tan); 7(Rab); 12, 21(Aga); 23(Tan à Aga); 38(Tet, Ksg); 40(Moh)

Ectocarpus Lyngbye

E. siliculosus (Dillwyn) Lyngbye

= E. confervoides Le Jolis

=E. fasciculatus Harvey

a) 1(Elj); 7, 16(Rab); 12, 21(Rab, Aga); 23(Atl); 28, 32(Nad); 31(Alh, Nad, Mel); 38(Tet, Ksg); 40(Moh); 48(Sal)

E. siliculosus (Dillwyn) Lyngbye var. crouanii (Thuret) Gallardo

$=\mathrm{E}$. crouanii Thuret

a) 38 (Tet, Ksg)

E. terminalis (Linnaeus) Nees
a) 4, 35(Tan); 40(Moh)

Feldmannia Hamel

F. battersiides (Ercegovic) Cormaci et Furnari a) $31(\mathrm{Nad}, \mathrm{Mel})$

F. globifera (Kützing) Hamel

a) 12, 21(Aga); 28, 32(Nad); 31(Alh, Nad, Mel)

F. caespitula (J. Agardh) Knoepffler-Péguy var. lebelii (Areschoug ex Crouan frat.) Knoepffler-Péguy 
=F. lebelii (Areschoug ex Crouan frat.) Hamel

= Ectocarpus lebelii (Areschoug) Crouan

a) 4, 35(Tan); 12, 21(Aga); 38(Tet, Ksg)

F. irregularis (Kützing) Hamel =Ectocarpus irregularis (Kützing) Hamel a) 4, 35(Tan); 21(cas); 28, $32(\mathrm{Nad})$; 31(Med); 38(Tet); 40(Moh);

F. paradoxa (Montagne) Hamel =Ectocarpus paradoxus Montagne

a) 38 (Tet, Ksg)

F. ralfsiae Vickers

a) 12, 21(Rab, Cas)

F. simplex ( Crouan frat.) Hamel =Ectocarpus simplex Crouan

a) 38(Tet, Ksg); 40(Moh)

Herponema (J. Agardh) Hamel

H. valiantei (Bornet) Hamel

a) $12,21(\mathrm{Elj})$

Hincksia J. E. Gray

H. granulosa (Smith) Silva

= Giffordia granulosa (Smith) Hamel

= Ectocarpus granulosus (Agardh) Sauvageau

a) 2(Saf, Ess); 4, 35(Tan); 12, 21(Rab); 38(Tet;Ksg); 40(Moh)

H. hincksiae (Harvey) Silva = Giffordia hincksiae (Harvey) Hamel

a) 2(Saf, Ess); 4, 35(Tan); 7(Rab); 12, 21(Ess, Aga); 23(Atl)

H. mitchelliae (Harvey) Silva

= Giffordia mitchelliae (Harvey) Hamel

$=\mathrm{G}$. veriscens (Thuret) Hamel

$=$ Ectocarpus veriscens Thuret

a) 12, 21(Sal, Rab, Aga); 16(Rab); 28, 32(Nad); 31(Med) ; 38(Tet, Ksg)

H. ovata (Kjellman) Silva $=$ Giffordia ovata (Kjellman) Kylin

$=\mathrm{G}$. intermedia (Rosenvinge) Lund

a) 31 (Alh, Nad, Mel)
H. sandriana (Zanardini) Silva =Giffordia. sandriana (Zanardini) Hamel = Ectocarpus elegans Thuret

a) 38 (Tet, Ksg)

H. secunda (Kützing) Silva

= Giffordia secunda (Kützing) Batters

a) 4, 35(Tan); 12, 21(Cas)

\section{Pilinia Kützing}

$P$. rimosa Kützing

a) 12,21 (Cas, Ess)

Streblonema Pringsheim

S. deformans (P. Dangeard) Hamel

a) 12,21 (Ess)

S. parasiticum (Sauvageau) De Toni

=Entonema. parasiticum (Sauvageau.) Hamel

a) 12, 21(Cas)

\section{Ralfsiaceae Farlow}

\section{Mesospora Weber}

M. macrocarpa (J. Feldmann) Hartog $=$ M. mediterranea J. Feldmann

a) 31 (Med); 38(Tet)

Nemoderma Schousboe ex Bornet

N. tingitanum Schousboe ex Bornet

a) 4, 35(Tan); 12, 21( $\mathrm{Sal}, \mathrm{Cas}) ; 31(\mathrm{Nad}, \mathrm{Mel})$

\section{Ralfsia Berkeley}

R. verrucosa (Areschoug) J. Agardh

a) 2(Saf, Ess); 7, 16(Rab); 12, 21(Cas, Elj); 31(Alh, Nad, Mel); 38(Tet, Ksg); 48(Sal)

\section{Fucales Kylin}

\section{Cystoseiraceae Kützing}

Bifurcaria Stackhouse

B. bifurcata (Velley) Ross

$=$ B. tuberculata Stackhouse

a) 1, 17(Elj); 2(Saf, Ess); 4, 35(Tan); 7, 11(Rab); 12, 21(Rab à Aga); 22(Ess); 27(Ess, Aga); 40(Moh); 48(Sal); 49, 50 (Rab, Cas); (Tan)* 
Cystoseira C. Agardh

C. abies-marina (S. G. Gmelin) C. Agardh ${ }^{(2)}$ a) 21 (Rab, Cas)

C. amentacea Bory

= C. selaginoides Naccari var. stricta Montagne

= C. stricta (Montagne) Sauvageau.

a) 30,31 (Alh, Nad;Mel)

C. baccata (Gmelin) Silva = C. fibrosa (Hudson) C.Agardh

a) 1(Elj); 4, 35(Tan); 7, 11(Rab); 12, 21(Rab à Aga); 22(Ess); 23(Atl); 30(Ich); 48(Sal); 50

C. brachycarpa J. Agardh var. balearica (Sauvageau) Giaccone

= C. balearica Sauvageau

= C. caespitosa Sauvageau

a) $30,31(\mathrm{Alh}, \mathrm{Nad}, \mathrm{Mel})$

C. compressa (Esper) Gerloff et Nizamuddin $=\mathrm{C}$. fimbriata (Desfontaines) Bory

= C. abrotanifolia C. Agardh

a) 1(Elj); 4, 35(Tan) 7, 16(Rab); 12, 21(Rab

à Ess); 23; 28, 32(Nad); 29(Alh, Nad); 30(Alh, Nad, Mel);31(Med); 38(Tet); 40(Moh)

C. crinita (Desfontaines) Bory

a) 28, 32(Nad); 30, 31(Alh, Nad, Mel)

C. elegans Sauvageau

a) 30,31 (Alh, Nad, Mel)

C. foeniculacea(Linnaeus) Greville

a) 12 (Sal, Cas;Elj), 21(Cas, Elj); 30; 52(Ceu, Mel)

C. humilis Kützing

a) 4, 35(Tan); 11, 12(Lar, Aga); 21(Elj); 30, 31(Alh, Nad, Mel); 50

C.humilis Kützing var. myriophylloides (Sauvageau) Giaccone $=$ C. myriophylloïdes (Sauvageau) Price et Jhon a) 1, 17(Elj); 7, 16(Rab); 11; 12, 21(Rab à Aga); 23; 40(Moh); 50

C. mauritanica Sauvageau

=C. selaginoides Valiante var. gibraltarica
Sauvageau

= C. gibraltarica Seauvageau

a) 7(Rab); 12, 21 (Rab, Elj); 23(Tan à Elj); 30, 31(Alh, Nad, Mel); 38(Ksg); 47(Tan)

C. mediterranea Sauvageau

a) 7, 12, 21, 35(Rab); 23(Saf); 30(Alh, NAd, Mel); 31(Nad, ICh); 32(Nad)

C. nodicaulis (Withering) Roberts = C. granulata (Turner) C. Agardh

a) $12,21,35$ (Ess); 23(Atl)

C. sauvageauana Hamel $=$ C. selaginoides Valiante

a) 4, 35(Elj); 22; 30, 31(Alh, Nad, Mel); 50

C. schiffneri Hamel

= C. discors (Linnaeus) C. Agardh

$=\mathrm{C}$. ercegovicii Giaccone

a) 12,21(Cas, Elj);30,31(Nad, Mel);35;48(Tan)

C. spinosa (Sauvageau) Montagne = C. montagnei Montagne

a) $30(\mathrm{Alh}, \mathrm{Nad}, \mathrm{Mel}) ; 31(\mathrm{Nad}, \mathrm{Mel}, \mathrm{ICh})$

C. tamariscifolia (Hudson) Papenfuss $=\mathrm{C}$. ericoides (Linnaeus) C. Agardh

a) 1, 17(Elj); 2(Saf, Ess); 7, 16(Rab); 12, 21 (Rab à Ess); 23(Atl, Med); 30(Alh, Nad, Mel); 31(Med); 38(Tet, Ksg); 40(Moh); 48(Sal); 50

C. usneoides (Linnaeus) Roberts

= C. platyclada Sauvageau

$=\mathrm{C}$. concatenata $\mathrm{C}$. Agardh

= Phyllacanta concatenata Kützing

a) 4, 35(Tan); 15, 48(Mel); 21(Cas, Elj); 30, 31(Alh, Nad, Mel); 50

C. zosteroides (Turner) J. Agardh = C. opuntiodes Bory

a) $30(\mathrm{Nad}, \mathrm{Mel}) ; 31(\mathrm{Nad})$

Fucaceae Adanson

Fucus Linnaeus ${ }^{(6)}$

Fucus spiralis Linnaeus

a) $1,17(\mathrm{Elj}) ; 2$ (Saf, Ess); 4, 35(Tan); 16(Rab); 22(Ess); 23(Atl); 27(Ess); 30(Alh, 
Nad, Mel); 31(Ceu, Alh, Nad); 38(Tet, $\mathrm{Ksg}$ ); 40(Moh); 48(Sal); 50(Cas)

Fucus spiralis Linnaeus var. platycarpus (Thuret) Batters

$=\mathrm{F}$. platycarpus Thuret

a) 4(Tan); 7(Rab); 11, 12(Rab, Aga); 49(Elj, Saf, Aga)

F. vesiculosus Linnaeus

a) 1(Elj); 12, 21(Rab à Ken); 23; 31(Nad); 35 (Ken à Elj)

F. vesiculosus Linnaeus var. axillaris (J.Agardh) Mangenot

$=\mathrm{F}$. axillaris J. Agardh

a) 1(Elj), 4, 35(Tan); 12, 21(Rab, Elj, Saf);

23(Sal, Elj); 48(Sal); (Ksg)*

F. vesiculosus Linnaeus var. lutarius Chauvin $=\mathrm{F}$. lutarius Kützing

a) 1(Elj); 35(Sal); 12, 21, 23(Sal à Cas)

\section{Sargasaceae Kützing}

Sargassum C. Agardh

S. acinarium (Linnaeus) C. Agardh $=\mathrm{S}$. linifolium (Turner) C. Agardh $=$ S. obtusatum Bory

a) $21 ; 30(\mathrm{Alh}, \mathrm{Nad}, \mathrm{Mel}) ; 31,32(\mathrm{Nad})$

S. desfontainesii (Turner) C. Agardh a) 29(Alh, Nad); 30(Alh, Nad, Mel); 31(Nad)

S. hornschuchii C. Agardh

a) 30 (Alh, Nad, Mel); 31(Med)

S. trichocarpum J. Agardh ${ }^{(5)}$

a) 4,35 (Tan)

S. vulgare C. Agardh

a) 1(Elj); 4, 35(Tan); 7, 16(Rab); 11, 12, 21(Rab, Elj); 23; 28, 29, 32(Nad); 30(Alh, Nad, Mel); 31(Med); 38(Tet, Ksg); 40(Moh); 48(sal); 50

\section{Laminariales Kylin}

\section{Laminariaceae Bory}

Laminaria Lamouroux L. ochroleuca Pylaie
$=$ L. lejolisii Sauvageau

a) 1, 17(Elj); 2(Saf, Ess); 4, 35(Tan); 11; 12, 21(Elj, Aga); 22(Ess); 23, 44(Elj, Ess); 50

L. rodriguezii Bornet

a) $15,35(\mathrm{Nad}) ; 30,52(\mathrm{Mel})$

\section{Sacchoriza Pylaie}

S.polyschides (Lightfoot) Batters = S. bulbosa (Hudson) Pylaie

a) 1, 17(Elj); 2(Saf, Ess); 4, 35(Tan); 7(Rab); 11; 12, 21(Lar à Aga); 22, 23, 44(Elj, Ess); 30(Alh, Nad, Mel); 31(Ceu, Alh, Nad, Mel); 49(Rab, Moh); 50; (Tan)*; (Saf)*

\section{Phyllariaceae Tilden}

Phyllariopsis Henry et South

P. brevipes (C. Agardh) Henry et South

= Phyllaria reniformis (Lamouroux) Rostafinsky

a) 4, 35(Tan); 11, 12, 21(Cas, Aga); 17(Elj); 30(Alh, Nad, Mel); 31 (Ceu, Alh, Nad, Mel); $50 ; 52$

P. purpurascens (C. Agardh) Henry et South $=$ Phyllaria purpurascens (C. Agardh) Rostafinsky

a) 4, 35(Tan); 12, 21(Cas, Ess, Aga); 17(Elj); 30, 33( Nad, Mel)

\section{Scytosiphonales Feldmann}

\section{Scytosiphonaceae Farlow}

Colpomenia (Endlicher) Derbés et Solier C. peregrina (Sauvageau) Hamel

a) 12, 21(Aga); 31(Alh, Nad)

C. sinuosa (Mertens ex Roth) Derbés et Solier a) 1(Elj); 4, 35(Tan); 7(Rab);12, 21(Rab, Cas Aga); 23(Atl, Med); 27(Aga); 28, 32(Nad); 31(Med); 38(Tet, Ksg); 40(Moh); 48(sal) 
Hydroclathrus Bory

H. clathratus (Bory) Howe

a) 27 (Aga)

Petalonia Derbés et Solier

P. fascia ( Müller) Kuntze

= Laminaria debilis C. Agardh

a) 1(Elj); 7(Rab); 23(Atl, Med); 31(Alh, Nad, Mel); 38(Tet, Ksg)

Scytosiphon C. Agardh

S. simplicissimus (Clemente) Cremades $=\mathrm{S}$. lomentaria (Lyngbye) Link

a) 4, 35(Tan); 28, 32(Nad); 31(Alh, Nad, Mel); 38(Tet, Ksg); 40(Moh)

\section{Sphacelariales Oltmanns}

\section{Cladostephaceae Oltmanns}

Cladostephus C. Agardh

C. spongiosus (Hudson) C. Agardh

$=\mathrm{C}$. verticillatus (Lightfoot) C. Agardh

$=\mathrm{C}$. hirsutus (Linnaeus) Boudouresque et Perret ex Boudouresque et al.

a) 1(Elj); 2(Saf, Ess); 4, 35(Tan); 7(Rab); 12, 21(Rab, Aga); 23(Atl, Med); 31(Med); 38(Tet, Ksg); 40(Moh); 48(Sal)

Discosporangium Falkenberg

D. mesarthrocarpum (Meneghini) Hauck

a) 31 (Ich)

Sphacelariaceae Decaisne emend. Oltmanns

Sphacelaria Lyngbye

S. brachygonia Montagne

a) 2(Saf, Ess); 4, 35(Tan); 31(Nad, Mel)

S. cirrosa (Roth) C. Agardh

$=$ S. hystrix Suhr ex Reinke

a) 4, 35(Tan); 12, 21(Rab, Cas); 31(Med); 38(Tet, Ksg); 40(Moh)

S. fusca (Hudson) S. F. Gray

a) 1(Elj); 2(Saf, Ess); 7(Rab); 23(Atl); 28,

32(Nad); 31(Med); 38(Tet, Ksg)

S. plumula Zanardini

a) 31 (Alh)
S. radicans (Dillwyn) C. Agardh

a) $12,21(\mathrm{Elj})$

S. rigidula Kützing

$=\mathrm{S}$. furcigera Kützing

a) 2(Saf, Ess); 4, 35(Tan); 28, 32(Nad);

31(Med); 38(Tet)

S. tribuloides Meneghini

a) 12, 21(Aga); 31 (Alh, Nad, Mel); 38(Tet); 40(Moh)

\section{Stypocaulaceae Oltmanns}

Halopteris Kützing

H. filicina (Grateloup) Kützing

a) 1(Elj); 4, 35(Tan); 7(Rab); 12, 21(Rab, Moh); 23(Atl, Med); 28, 32(Nad); 31(Med); 38(Tet, Ksg); 40(Moh); 48(Sal)

H. scoparia (Linnaeus) Sauvageau = Stypocaulon scoparium (Linnaeus) Kützing

a) 1(Elj); 2(Saf, Ess); 4, 35(Tan); 7(Rab); 12, 21(Rab à Elj); 23(Atl, Med); 28, 32(Nad); 31(Med); 38(Tet, Ksg); 40(Moh); $48(\mathrm{Sal})$

\section{Sporochnales Sauvageau (2)}

\section{Sporochnaceae Greville}

\section{Carpomitra Kützing}

C. costata (Stachouse) Batters Var. mediterranea J. Feldmann

$=$ Sporochnus dichotomus Zanardini

a) 4,35 (Tan)

Nereia Zanardini

N. filiformis (J. Agardh) Zanardini

a) 4,35 (Tan)

Sporochnus C. Agardh

S. gaertneri (Gmelin) C. Agardh a) 4,35 (Tan)

S. pedunculatus (Hudson) C. Agardh a) 4,35 (Tan) 


\section{Liste Alphabétique des Taxa}

Les chiffres romains indiquent le numero des Ordres relatif à chaque Classe:

$\mathrm{C}=$ Chlorophyceae, $\mathrm{P}=$ Phaeophyceae .

Acetabularia acetabulum (IVC)

A. calyculus

A. mediterranea

A. moebii

A. parvula

Acinetospora crinita (VIP)

A. pusilla

A. vidovichii

Aglaozonia melanoidea (IIP)

A. parvula

Asperococcus bullosus (IVP)

A. compressus

A. turneri

Bachelotia fulvescens (VIP)

Bifurcaria bifurcata (VIIP)

B. tuberculata

Blidingia marginata (VIC)

B. minima

Bryopsidella neglecta (IIC)

Bryopsis adriatica (IIC)

B. balbisiana

B. corymbosa

B. cupressina var. adriatica

B. cupressoides

B. disticha

B. duplex

B. feldmannii

B. hypnoides

B. monoica

B. muscosa

B. pennata

B. plumosa

Carpomitra costata var. mediterranea (XIP)

Caulerpa prolifera (IIC)

C. racemosa

Chaetomorpha aerea (IIIC)

C. capillaris

C. gracilis

C. linum

C. mediterranea

C. pachynema

Cladophora albida (IIIC)

C. coelothrix
C. dalmatica

C. echinus

C. globulina

C. hamosa

C. hirta

C. hutchinsiae

C. laetevirens

C. lehmanniana

C. pellucida

C. prolifera

C. punica

C. ramosissima

C. ramulosa

C. rudolphiana

C. rupens

C. rupestris

C. sericea

C. utriculosa

C. vadorum

C. vagabunda

Cladophoropsis membranaceus (IIIC)

Cladostephus hirsutus (XP)

C. spongiosus

C. verticillatus

Codium adhaerens (IIC)

C. bursa

C. decorticatum

C. difforme

C. effusum

C. elongatum

C. fragile

C. tomentosum

C. vermilara

Colpomenia peregrina (IXP)

C. sinuosa

Cutleria adspersa (IIP)

C. multifida

Cystoseira abies-marina (VIIP)

C. abrotanifolia

C. amentacea

C. baccata

C. balearica

C. brachycarpa var. balearica

C. caespitosa

C. compressa

C. concatenata

C. crinita

C. discors

C. elegans

C. ercegovicii 
C. ericoides

C. fibrosa

C. fimbriata

C. foeniculacea

C. gibraltarica

C. granulata

C. humilis

C. humilis var. myriophylloides

C. mauritanica

C. mediterranea

C. montagnei

C. myriophylloides

C. nodicaulis

C. opuntioides

C. platyclada

C. sauvageauana

C. schiffneri

C. selaginoides

C. selaginoides var. gibraltarica

C. selaginoides var. stricta

C. spinosa

C. stricta

C. tamariscifollia

C. usneoides

C. zosteroides

Dasycladus clavaeformis (IVC)

D. vermicularis

Derbesia lamourouxii (IIC)

D. neglecta

D. tennuissima

Desmarestia ligulata (IIIP)

D. tingitana

Dictyopteris membranacea (VP)

D. polypodioides

Dictyota dichotoma (VP)

D. divaricata

D. linearis

Dilophus fasciola (VP)

D. ligulatus

D. spiralis

Discosporangium mesarthrocarpum (XP)

Ectocarpus confervoides (VIP)

E. crouanii

E. elegans

E. fasciculatus

E. granulosus

E. irregularis

E. lebelii

E. paradoxus

E. siliculosus
E. siliculosus var. crouanii

E. simplex

E. terminalis

E. virescens

Elachista flaccida (IP)

E. fucicola

Endoderma viride (VC)

Enteromorpha clathrata (VIC)

E. crinita

Enteromorpha compressa

E. fasciculata

E. flabellata

E. flexuosa

E. intestinalis

E. kylinii

E. lingulata

E. linza

E. marginata

E. multiramosa

E. muscoides

E. prolifera

E. ramulosa

E. scopulorum

E. simplex

E. torta

E. tuberculosa

Enthocladia viridis (VC)

Entonema parasiticum (VIP)

Feldmannia battersiides (VIP)

F. caespitula var. lebelii

F. globifera

F. irregularis

F. lebelii

F. paradoxa

F. ralfsiae

F. simplex

Flabellia petiolata (IIC)

Fucus axillaris (VIIP)

F. lutarius (VIIP)

F. platycarpus (VIIP)

F. spiralis (VIIP)

F. spiralis var. platycarpus

F. vesiculosus (VIIP)

$F$. vesiculosus var. axillaris

$F$. vesiculosus var. lutarius

Giffordia granulosa (VIP)

G. hinckisiae

G. intermedia

G. mitchelliae

G. ovata 
G. sandriana
G. secunda
G. veriscens

Gonidia rivulariae (IP)

Halicystis parvula (IIC)

Halimeda tuna (IIC)

Haloglossum compressum (IVP)

Halopteris filicina (XP)

H. scoparia

Herponema valiantei (VIP)

Hincksia granulosa (VIP)

H. hincksiae

H. mitchelliae

H. ovata

H. sandriana

H. secunda

Hydroclathrus clathratus IXP

Laminaria debilis (IXP)

L. lejolisii

L. ochroleuca (VIIIP)

L. rodriguezii

Leathesia difformis (IP)

L. marina

Leptonema fasciculatum (IP)

Leptonematella fasciculata (IP)

Liebmannia leveillei (IP)

Lithosiphon laminariae (IP)

Mesogloea griffithsiana (IP)

M. vermiculata

Mesospora macrocarpa (VIP)

M. mediterranea

Monostroma grevillei (VIC)

M. obscurum

M. oscycoccum

Myriactula rivulariae (IP)

Myrionema strangulans (IP)

M. vulgare

M. vulgare var. maculaeformis

Nemoderma tingitanum (VIP)

Nereia filiformis (XIP)

Padina pavonia (VP)

$P$. pavonica

Pedobesia lamourouxii (IIC)

P. solieri

Percursaria percusa (VIC)

Petalonia fascia (IXC)

Phaeophila dendroides (VC)

$P$. divaricata

$P$. viridis

Phyllacanta concatenata (VIIP)
Phyllaria reniformis (VIIIP)

$P$. purpurascens

Phyllariopsis brevipes (VIIIP)

$P$. purpurascens

Pilinia rimosa (VIP)

Polyphysa parvula (IVC)

Pringsheimiella scutata (VC)

Pseudochlorodesmis furcellata (IIC)

Pseudopringsheimia confluens (VC)

Ralfsia verrucosa (VIP)

Rhizoclonium kerneri (IIIC)

R. kochianum

R. riparium

R. tortuosum

Sacchoriza bulbosa (VIIIP)

S. polyschides

Sargassum acinarium (VIIP)

S. desfontainesii

S. hornschuchii

S. linifolium

S. obtusatum

S. trichocarpum

S. vulgare

Sauvageaugloia griffithsiana (IP)

Scytosiphon lomentaria (IXP)

S. simplicissimus

Spatoglossum areschougii (VP)

S. solierii

Sphacelaria brachygonia (XP)

S. cirrosa

S. furcigera

S. fusca

S. hystrix

S. plumula

$S$. radicans

S. rigidula

S. tribulloides

Sporochnus dichotomus (XIP)

S. gaertneri (XIP)

S. pedunculatus

Streblonema deformans (VIP)

S. parasiticum

Stypocaulon scoparium (XP)

Taonia atomaria (VP)

Udotea petiolata (IIC)

Ulothrix flacca (VC)

U. subflaccida

Ulva bifrons (VIC)

U. cribosa

U. curvata 


\author{
U. elegans \\ U. fasciata \\ U. lactuca \\ U. olivascens \\ $U$. rigida \\ U. schousboei \\ Ulvella confluens (VC) \\ $U$. setchellii \\ Ulvopsis grevillei (VIC) \\ Urospora leata (IC) \\ Valonia macrophysa (IIIC) \\ V. urticularis \\ $V$. ventricosa \\ Ventricaria ventricosa (IIIC) \\ Zanardinia collaris (IIP) \\ Z. prototypus \\ Zonaria adspersa (IIP) \\ Z. dichotoma (VP) \\ Z. linearis \\ Z. tournefortii
}

\section{Notes}

1. Compte tenu des notes de Perret-Boudouresque et al., 1989, Gallardo et al. 1993 et Conde et al. 1994, basés sur les travaux de Bliding 1986, il n'est pas exclus, qu'une partie au moins des signalisations de cette espèce au Maroc se rapportent à des formes ou à des variétés particulières ou même à des Ulves désignées actuellement sous d'autres noms.

2. La présence doit être confirmée.

3. Il s'agit de deux générations d'une même espèce mais nous jugeons qu'il est nécessaire de respecter les citations telles qu'elles ont été publiées.

4. Même commentaire que (3)

5. Doit être réétudiée sur les sites signalés.

6. Compte tenu des données de Pérez Ruzafa \& Gallardo 1997 sur la côte Ibérique, les variétés , les formes et la répartition des Fucus doivent être réétudiées sur les côtes marocaines.

\section{BIBLIOGRAPHIE}

1. BERDAY, N. -1989- Contribution à l'étude écologique du phythobenthos de la Zone littorale de la région d'EL Jadida. Doctorat de 3ème cycle. Fac. Sc. Rabat.

2. BIRJE, J., M. VERLAQUE \& F. POYDENOT 1995- Macrophytobentos des platiers rocheux intertidaux et semi-exposées de la région de Safi-Essaouira (Maroc occidental). Oceanologica acta vol. 19- $\mathrm{n}^{\circ}$ 5: 561-574

3. BLIDING, C. -1986 - A critical survey of European taxa in Ulvales. II: Ulva, Ulvaria, Monostroma, Kormania. Bot. Notiser: 121:535629.

4. BORNET, E. -1892- Les algues de P.K.A Schousboe récoltées au Maroc et dans la Méditerranée, déterminées par M. Edouard Bornet de 1815 à 1829 extrait des mémoires de la Soc. Nat. et Math. de Cherbourg. Tome (XXVIII).

5. BOUDOURESQUE, C.F., M. PERRETBOUDOURESQUE \& M. KNOEPFFLERPEGUY -1984- Inventaire des algues marines benthiques dans les Pyrénées orientales (Méditerranée, France). Vie et milieu, Sér. A, 34 (1): 41-59.

6. BURROWS., E.M. -1991-Seaweed of the British Isles. 2. Chlorophyta. Natural Museum. London 238pp.

7. CAVASSILLAS, B. -1963-Étude morphologique, écologique et floristique du bassin D'El Harhoura . Mémoire Soc. Sc. Nat et Phy. du Maroc. Bot. (nouv. Ser.). $\mathrm{n}^{\circ} 3$.

8. CONDE POYALES, F. -1984- Contribución al conocimiento de la flora bentónica del Mar de Alborán. Islas Chafarinas. Acta Bot. Malacitana 9: 41-46.

9. CONDE POYALES, F. -1992- Sobre la colección de algas del herbario de la Sociedad Malagueña de Ciencias (S. XIX). Acta Bot. Malacitana 17: 29-55.

10. DANGEARD, P. -1935-Sur l'identité de Laminaria lejolisii Sauv. et Laminaria iberica (Ham.) Lam. C. R. Acad. Sci., 201, p: 615.

11. DANGEARD, P. -1937- Sur les zones d'algues marines du Maroc occidental. C. R. Acad. Sci., 204, p: 795.

12. DANGEARD, P. -1949- Les algues marines de la côte occidentale du Maroc. Le Botaniste $\mathrm{n}^{\circ}$ 34: 89-189.

13. DANGEARD, P. -1957- Observations écologiques sur les algues du Maroc atlantique. Colloques Internationaux du C.N.R.S. 
14. DEBRAY, F. -1897- Catalogue des algues du Maroc, d'Algerie et de la Tunisie. A. Jourdan Edit. Alger., 78pp.

15. DE BUEN, O. -1913- Notes sur les fonds et sur la pêche dans la côte méditerranéenne du Rif. Atti V Congrs. Internaz. di Pesca. Roma.

16. ESMIOLE, J.P. -1962- Recherche sur la végétation marine d'une zone littorale au sud de Rabat. Trav. I. Sc. Sér. Bot. no 25.

17. FELDMANN, J. -1955- La Zonation des algues sur la Côte atlantique du Maroc.Bull. Soc. Nat. et Physique 35, 1er trimestre. Maroc. 9-18.

18. FLORES -MOYA, A., J. SOTO, A. SÁNCHEZ, M. ALTAMIRANO, G. REYES \& F. CONDE. 1995a - Check-list of Andalusia (S.SPAIN) seaweed.I. Phaeophyceae

Acta Bot. Malacitana, 20: 5-18.

19. FLORES -MOYA, A., J. SOTO, A. SÁNCHEZ, M. ALTAMIRANO, G. REYES \& F. CONDE. 1995b - Chech-list of Andalusia (S.SPAIN) seaweed.II. Chlorophyceae Acta Bot. Malacitana, 20: 19-26.

20. GALlARDO, T., A. GÓMEZ GARRETA, M. A. RIBERA, M. CORMACI, G. FURNARI, G. GIACCONE \& C. F. BOUDOURESQUE - 1993 Chek-list of Mediterranean Seaweed II. Chlorophyceae Wille, s.l. Botanica Marina 36: 399-421.

21. GATTEFOSSE \& WERNER, R. G. \& J. -1935Catalogus algarum Marocanorum adhuc cognitorum. Bull. Soc. Sc. Nat. et Phys. du Maroc. 72-107.

22. GAYRAL, P. -1957- Résultat d'une prospection algologique à l'île de Mogador. C. R. Acad. Sci. Vl. 245, p: 358-360.

23. GAYRAL, P. -1958-Algues de la côte atlantique marocaine. La nature au Maroc, II. 523p.

24. GAYRAL, P. \& R. AURO -1958- Observation sur les variations morphologiques d'une zone côtière adjacente à l'estuaire de Bouregreg (Rive gauche). Bull. Soc. Nat. et Phy. du Maroc. 38, (2): 59-70.

25. GAYRAL, P. -1961- Liste commentée des algues marines nouvelles pour le Maroc reconnues depuis 1949. Bull. Soc. Sc. Nat. et Phy. du Maroc $\mathrm{n}^{\circ}$ 41. pp: 1-18.

26. GAYRAL, P. -1966- Les algues des côtes françaises (Manche, Atlantique). Edition Doin, Paris.

27. GIL-RODRÍGUEZ, M. C. \& J. S. SOCORRO
HERNÁNDEZ - 1986- Notas ficológicas acerca de la costa atlántica-marroqui. Vieraea 16: 193 198.

28. GONZÁlEZ GARCÍA, J. A. \& F. CONDE 1991- Estudio florístico, fenológico, autoecológico y fitogeográfico del macrofitobenthos de la Mar Chica (Sebcha Buareg de Nador, Mediterráneo Marroqui) . Acta Bot. Malacitana 16 (1): 63-80.

29. GONZÁlEZ GARCÍA, J. A. \& F. CONDE 1992- Sargassum desfontainesii (Turner) C. Agardh (Fucales, Fucophyceae), primera cita para el Mediterráneo. Acta Bot. Malacitana 17: 250-251.

30. GONZÁlEZ GARCÍA, J. A. \& F. CONDE 1993- Estudio biogeográfico de las Fucales y Laminariales atlánticas en el littoral mediterráneo de Marruecos. Acta Bot. Malacitana 17: 3944.

31. GONZÁlEZ GARCÍA, J. A. \& F. CONDE 1994- Catalogo del macrofitobenthos del Mediterráneo de Marruecos. Acta. Bot. Malacitana 19: 5-27.

32. GONZÁLEZ GARCÍA, J. A. \& F. CONDE 1995- Etude comparative sur le macrophytobenthos de trois lagunes côtières de la Méditerrannée occidentale Nova Hedwigia 61. 3-4 pp 377-390 Stuttgart.

33. GONZÁLEZ GARCÍA, J. A. \& F. CONDE 1995- Sobre la presencia de Phyllariopsis purpurascens (C. Agardh) Henry \& South en el Mediterráneo de Marruecos. Acta Bot. Malacitana 20 p 283-284

34. HAMEL, G. -1930-Chlorophycées des côtes Françaises. Rouen: 224p.

35. HAMEL, G. -1939- Phéophycées des côtes Françaises. Paris: $429 \mathrm{p}$

36. HARIOT, M.P. -1909- Sur une collection d'algues recueillies au Maroc par M. Buchet. Bull. Mus. Hist. Nat. 3: 128-130.

37. HARIOT, M.P. -1911- Sur une collection d'algues recueillies en Mauritanie par M. Chudeau. Bull. Soc. Bot. Fr. 58: 438-445.

38. KAZZAZ, M. -1989-Contribution à l'étude de la flore algale de la région ouest de la Méditerranée, Doctorat de 3 ème cycle. Fac. des Sc. Rabat 246p.

39. LOZANO CABO, F. -1953-. Notas sobre una prospección pesquera en la Mar Chica.Bol. Inst.Esp.Ocean. 64:3-37. 
40. OUAHI, M. -1987- Etude quantitative et qualitative des algues du littoral de Mohammédia. D.E S. Fac. des Sciences Rabat.

41. PERES J. M. \& J. PICARD -1964- Nouveau manuel de bionomie benthique de la mer Méditerranée. Rec. Trav. Stat. Mar. Endoume 31 (47): 5-137.

42. PÉREZ-RUZAFA, I. M. \& T. GALLARDO 1997- Mapas de distribución de algas de la Península Ibérica e Islas Baleares. X. Variedades de Fucus spiralis L. y de F. vesiculosus L. (Fucales, Fucophyceae). Botanica Complutensis, 21: 121-136

43. PERRET-BOUDOURESQUE, M. \& H. SERIDI -1989-Inventaire des algues marines benthiques d'Algérie. GIS. Posidonie publ., Marseille, Fr., 1-117.

44. RIADI, H. -1989- Etude des algues benthiques productrices d'hydrocolloüdes (Laminaria ochroleuca et Gelidium sesquipedale). Possibilité de commercialisation de l'acide alginique, Doctorat de 3 ème cycle Fac. des Sc. Rabat

45. RIBERA, M. A., A. GÓMEZ-GARrETA, T. GALLARDO, M. CORMACI, G. FURNARI \& G. GIACCONE -1992- Chek-list of mediterranean seaweed I. Fucophyceae (Warming 1984). Botanica Marina 35: 109130.

46. SALAHDDINE, N. V. -1982-Etude quantitative et qualitative du macrophytobenthos d'un secteur (Rabat-Bouznika) du littoral océanique marocain. Mém. de 2 ème cycle, I. A.V., Hassan II Rabat.

47. SAUVAGEAU, C. -1913- Sur les Fucacées du Détroit de Gibraltar. Comp. Rend. Hebd. Séances Acad. Sc., 157: 1539-1540

48. TAHI, M. -1983-Etude qualitative et quantitative du macrophytobenthos d'un secteur (Salé-Bouknadel) du littoral atlantique marocain. Mém. de fin d'étude, option halieutque I. N. A. V: Hassan II Rabat.

49. WERNER, R.G. -1948- Observation sur la répartition des algues et en particulier les Laminaires au Maroc. Bull. Soc. Sc. Nat. et Phy. du Maroc. $\mathrm{n}^{\circ}$ 6: 56-58.

50. WERNER, R.G. -1955-Contribution à l'étude de la flore cryptogamique au Maroc. Bull. Soc. Nat et Phy. du Maroc: 19-68.
51. WERNER, R.G. -1956- Réflexion au sujet de la zonation des algues au Maroc. Bull. Soc. Nat et Phy. du Maroc. $\mathrm{n}^{\circ}$ 36: 319-334.

52. WERNER, R. G. -1962- Essai d'une étude de la répartition des cryptogames marines et maritimes du Maroc. Bull. Soc. Nat et Phy. du Maroc. $\mathrm{n}^{\circ}$ 42: 1-33

Aceptado para su publicación en Junio de 1998

Adresse des auteurs. Groupe d'Ecologie et Biologie des Populations (GEBP). Département de Biologie. Faculté des Sciences de Tetouan BP. 2121, Tétouan 